\title{
1 Characterization and simulation of metagenomic nanopore sequencing data
}

\section{2 with Meta-NanoSim}

3 Chen Yang $^{1,2}$, Theodora Lo $^{1,2}$, Ka Ming $\mathrm{Nip}^{1,2}$, Saber Hafezqorani ${ }^{1,2}$, Rene L Warren ${ }^{1}$, Inanc

4 Birol $^{1,3^{*}}$

5 1. Canada's Michael Smith Genome Sciences Centre, BC Cancer, Vancouver, BC, Canada

6 2. Bioinformatics Graduate Program, University of British Columbia, Vancouver, BC, Canada

7 3. Department of Medical Genetics, University of British Columbia, Vancouver, BC, Canada

$8 *$ Corresponding author

10 ABSTRACT

11 Nanopore sequencing is crucial to metagenomic studies as its kilobase-long reads can

12 contribute to resolving genomic structural differences among microbes. However, platform-

13 specific challenges, including high base-call error rate, non-uniform read lengths, and the

14 presence of chimeric artifacts, necessitate specifically designed analytical tools. Here, we

15 present Meta-NanoSim, a fast and versatile utility that characterizes and simulates the unique

16 properties of nanopore metagenomic reads. Further, Meta-NanoSim improves upon state-of-

17 the-art methods on microbial abundance estimation through a base-level quantification

18 algorithm. We demonstrate that Meta-NanoSim simulated data can facilitate the development

19 of metagenomic algorithms and guide experimental design through a metagenomic assembly

20 benchmarking task. 
23 Metagenomics, Oxford nanopore sequencing, microbial abundance estimation, sequence

24 simulation

\section{BACKGROUND}

27 Empowered by the rapid development of next-generation sequencing technologies,

28 metagenomic analysis has enabled comprehensive investigation of the genetic composition and

29 abundance of microbial communities. In investigating microbiomes, metagenomic sequencing

30 bypasses the need to culture each individual species by extracting DNA directly from their

31 natural habitat, making it feasible to study the microbes that cannot be isolated or cultured in

32 laboratory $(1,2)$. Within the past few decades, the improved throughput and reduced cost of

33 next-generation sequencing platforms have enabled a wide range of metagenomic studies of

34 environmental, pharmaceutical, and medical relevance (3-5).

36 Until recently, Illumina short-read sequencing (Illumina Inc., San Diego, CA) has been the

37 technology of choice for metagenomic sequencing projects due to its high throughput, low cost,

38 and low error rate. However, the reads generated by Illumina instruments are often too short

$39(<250 \mathrm{bp})$ to span inter- and intra-chromosomal homologous regions and suffer from intrinsic

40 biases, thus complicating downstream assembly and taxonomic analysis (6). As a third-

41 generation long-read sequencing technology from Oxford Nanopore Technologies Ltd. (ONT,

42 Oxford, UK), nanopore sequencing is gaining traction in metagenomic research efforts, due

43 largely to the long read lengths it generates, as well as the portability of their MinION

44 sequencing platform (7). The N50 (the shortest read length to be included for covering $50 \%$ of 
45 the total sequenced length) in a typical run is over $5 \mathrm{~kb}(8)$ and the reported maximum read

46 length exceeds $2 \mathrm{Mb}$. At the high end, whole bacterial or viral genomes may be captured by a

47 few sequencing reads $(9,10)$, making it possible to disambiguate between even closely-related

48 strains. Since it was first announced, metagenomic sequencing by ONT has been playing an

49 essential role in real-time pathogen identification and clinical diagnosis, including during the

50 recent coronavirus disease 2019 pandemic (11-14).

51

52 Although a plethora of metagenomic analysis tools have been developed for short-read

53 sequencing data, the challenges associated with ONT reads, such as high error rate, non-

54 uniform error distributions, and chimeric read artifacts $(8,15-17)$, are calling for analytical tools

55 designed specifically for long reads. For example, quantification of microbial abundance levels,

56 or metagenomic abundance estimation, is traditionally computed by counting the number of

57 mapped reads followed by fine-tuning of ambiguous mappings $(18,19)$. This approach has been

58 proven to be cost-effective for Illumina short reads because of their uniform lengths. However,

59 the accuracy of these tools would be understandably impacted when applied on ONT reads,

60 especially for lowly represented genomes, because of the variable lengths and relatively high

61 error rates compared to Illumina reads (5 - 15\% depending on the flowcell chemistry and

62 basecalling algorithm). In addition, ONT sequencing projects on genome, transcriptome, and

63 metagenome, from prokaryotes to eukaryotes, were all reported to have certain problematic

64 reads with gapped or chimeric alignments, likely generated due to library preparation or

65 sequencing artifacts $(17,20-25)$. Reference-based abundance estimation using merely primary

66 alignments may further be affected by the presence of these chimeric reads, as well as reads 
67 that span the start position of a circular genome. To the best of our knowledge, even the state-

68 of-the-art program, MetaMaps, does not account for chimeric reads, but simply uses an

69 Expectation-Maximization (EM) algorithm to disambiguate multi-mapped reads (26). In this

70 work, we show that there is still room for improving metagenomic abundance estimation, a

71 proposition attainable by quantifying aligned bases instead of reads, while leveraging chimeric

72 read information.

73

74 In the process of tool development and benchmarking, a metagenomic ONT read simulator and

75 associated simulated datasets with known ground truth can save time and money. Ideally, such

76 a read simulator should reflect the true characteristics of the ONT platform and allow effective

77 evaluation of bioinformatics tools. In return, the evaluation results can guide the experimental

78 design of metagenomics project, such as sequencing depths and number of replicates (27).

79 Currently, the only simulator that specifically simulates ONT metagenomic datasets is CAMISIM

80 (28). The workflow of CAMISIM is focused on the composition design of microbial community

81 given a taxonomy profile. It uses NanoSim (version 1) (15) as the engine to simulate ONT reads

82 for each genome separately once the composition of the community is determined. Following

83 the same idea, one can also use other existing ONT genomic simulators naively to simulate each

84 composite genome separately and then aggregate the reads according to the desired

85 abundance. However, it is impractical to simulate a large microbial community with hundreds

86 or more genomes with this approach, not to mention the existing simulators for ONT reads are

87 not designed to model metagenomic specific features, such as chimeric reads and deviations in

88 abundance levels. More importantly, the simulation of abundance levels should be consistent 
89 with the quantification method, thus merely mixing the reads from different genomes will yield

90 a compromised abundance profile. Taken together, we believe the current version of NanoSim

91 can be upgraded to capture and simulate read properties specific to metagenomics, especially

92 the microbial abundance levels and chimeric reads - two key factors that may influence

93 metagenome assembly, taxonomy binning, and abundance estimation. Further, in real world

94 scenarios, viruses, bacteria, and fungi co-exist in complex microbial communities, hence it is a

95 desired feature to simulate metagenomes comprising both circular and linear genomes.

97 Here, we introduce Meta-NanoSim (NanoSim version 3), an ONT metagenome simulator for

98 complex microbial communities. Given a training dataset, Meta-NanoSim characterizes read

99 length distributions, error profiles, and alignment ratio models. Optionally, it also detects

100 chimeric reads and estimates microbial abundance levels. In our benchmarks, the performance

101 of the metagenomic abundance estimation feature of Meta-NanoSim surpasses the current

102 state-of-the-art methods. The chimeric read detection feature also improves upon the read

103 length modelling in NanoSim v2, and thus simulating this artifact of the technology may

104 challenge metagenomic analytical tools with a real-world scenario. Through benchmarking

105 experiments comparing simulated reads with empirical datasets, we show that Meta-NanoSim

106 preserves the key characteristics of ONT metagenomic reads. Further, we showcase the

107 usability and utility of Meta-NanoSim with a metagenomic assembly task.

108

\section{RESULTS}

110 Here, we first present the design of Meta-NanoSim and demonstrate the utility and 
111 performance of two key features, chimeric read detection and abundance estimation. To show

112 the similarity between simulated reads and experimental reads, we generated a simulated

113 dataset using models learned from experimental data and compared against a CAMISIM

114 simulated dataset. We also show that Meta-NanoSim is capable of simulating large complex

115 microbial community by simulating a dataset with 125 species with abundance levels estimated

116 from a human saliva sample. Finally, we showcase a potential use case of Meta-NanoSim with a

117 set of simulated data, benchmarking the metagenomic assembler MetaFlye (29) on how it

118 scales with increasing sequencing depth. The input experimental data used in this section are

119 two sets of publicly available mock community ONT sequencing reads, each dataset containing

120 the same 10 microbial species (eight bacteria and two fungi) but with different abundance

121 distributions. In this work, we denote the dataset with evenly distributed abundance levels as

122 the Even dataset and the one with logarithmically distributed abundance levels as the Log

123 dataset (detailed in Methods).

\section{Meta-NanoSim design}

126 Meta-NanoSim is implemented in Python as two sub-modules in the NanoSim suite: meta in

127 characterization and simulation stages. It learns the technical and metagenomic-specific

128 features of ONT reads in the characterization stage, builds statistical models, and applies them

129 in the simulation stage (Fig. 1). In the characterization stage, it takes ONT metagenomic reads

130 and a reference metagenome as input to infer the ground truth through sequence alignments.

131 Based on those alignments, it models the read length distributions (aligned and unaligned part)

132 via kernel density estimation and basecall events via mixture statistical models. In addition to 
133 existing NanoSim features, we introduce two new analyses in its characterization pipeline;

134 chimeric reads analysis for genome/metagenomes and abundance estimation for metagenomic

135 datasets (Methods).

137 To simulate a metagenomic dataset, besides the pre-trained model from the characterization

138 stage, a list of reference genomes of users' choice is required as input to use for simulation,

139 together with their abundance levels and DNA topology (i.e. linear or circular). The tool can also

140 stream reference genome sequences from either RefSeq (30) or Ensembl (31) automatically

141 without requiring extra disk storage, which facilitates large microbial community simulations.

142 Since microbial sequencing projects are often carried out in a multi-sample or multi-replicate

143 fashion, Meta-Nanosim is designed to simulate multiple samples in one batch with user-defined

144 abundance level profiles as input.

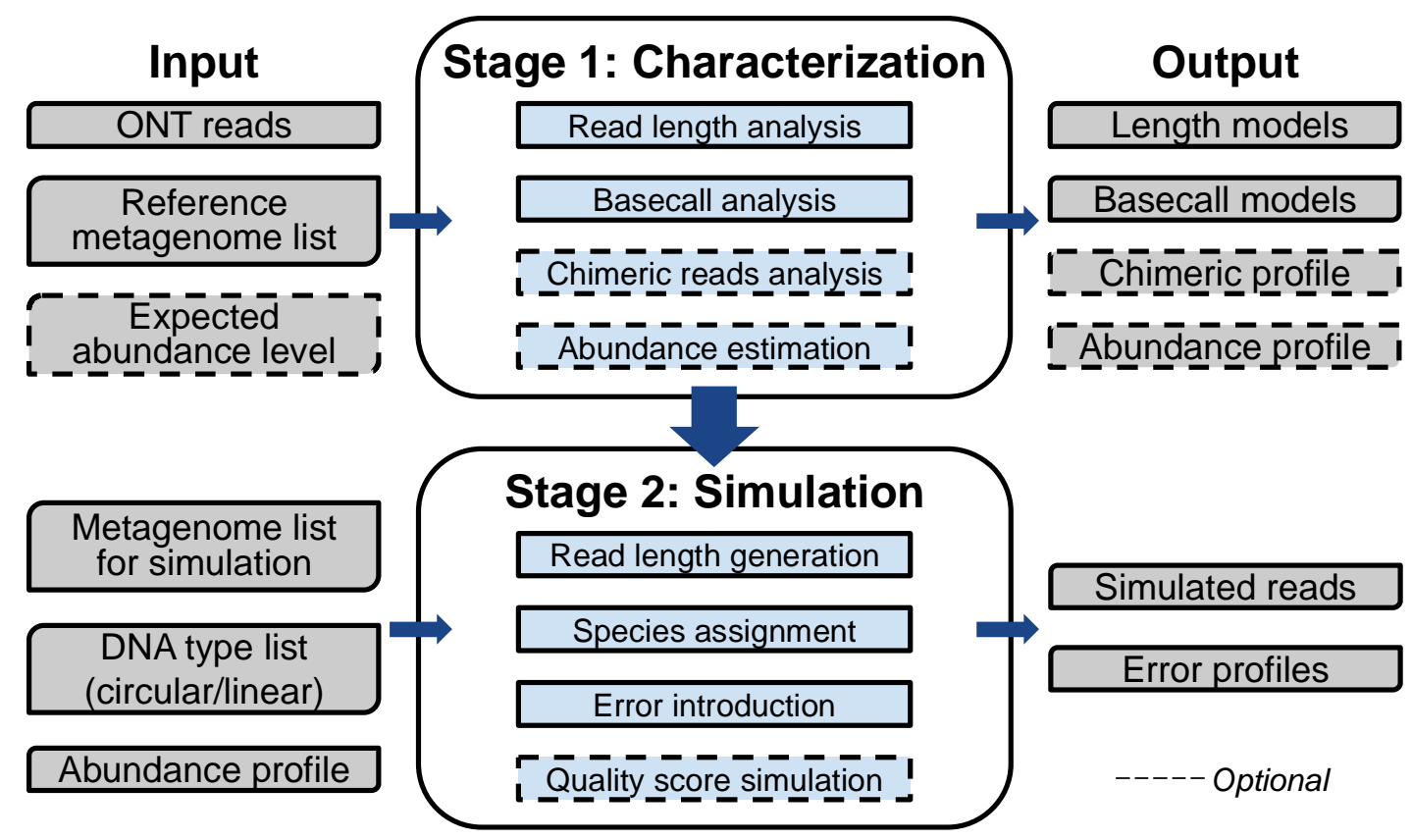

Fig. 1 Meta-NanoSim workflow. Meta-NanoSim consists of two stages: characterization stage and simulation stage. In the characterization stage, given a training dataset and reference metagenome, Meta-NanoSim builds models for the read length distributions and basecall events. It optionally profiles 
chimeric read artifacts and quantifies an abundance profile. It can also calculate the deviation between expected and estimated abundance levels. In the simulation stage, Meta-NanoSim takes four inputs: 1) The genome list for simulation (local or ftp path), 2) DNA type list, 3) abundance profile for simulation, and 4) the models generated from the characterization stage. Meta-NanoSim outputs simulated reads and error profiles as ground truth.

146 Chimeric read characterization and simulation

147 Chimeric reads are also called split reads because one read is split into two or more sub-

148 alignments that are aligned to distinct regions of the genome / metagenome. They may be

149 created due to sequencing artifacts or structural variants when the reference metagenome is

150 not comprehensive. Previous studies reported that chimeric reads represent a non-negligible

151 fraction of ONT sequencing datasets ranging from $1.7 \%$ to $8.17 \%$ with different seuqncing kit

152 and identificaiton thresholds $(17,20,22,23)$. In the metagenome datasets used in our study,

153 after ruling out structural variants, we have identified a similar fraction of reads in this category:

$1542.17 \%(75,628$ reads) in the Even dataset and $1.67 \%(68,444$ reads $)$ in the $\log$ datasets. These

155 reads are free of known adapters, so their presence may impact downstream analyses, such as

156 assembly, taxonomy binning, and quantification, even after adapter trimming. When aligned to

157 their respective reference genome sequence(s), ONT reads may contain unaligned or soft-

158 clipped regions. In our tests, the chimeric read detection feature of Meta-NanoSim significantly

159 reduced the length of these unaligned regions, which explained why some of the reads have

160 over $1 \mathrm{kbp}$ long unaligned portions (Fig. 2A). As seen in Fig. 2B, the length distributions of the

161 gaps between split alignments follow multi-modal distributions. Meta-NanoSim uses kernel

162 density estimation to model them, with results exhibiting strong similarity between the length

163 distributions of simulated and experimental sequences. We also noticed that the number of

164 segments each read contains can be described as a geometric distribution and the mean 
bioRxiv preprint doi: https://doi.org/10.1101/2021.11.19.469328; this version posted November 20, 2021. The copyright holder for this preprint (which was not certified by peer review) is the author/funder, who has granted bioRxiv a license to display the preprint in perpetuity. It is made available under aCC-BY-NC 4.0 International license.
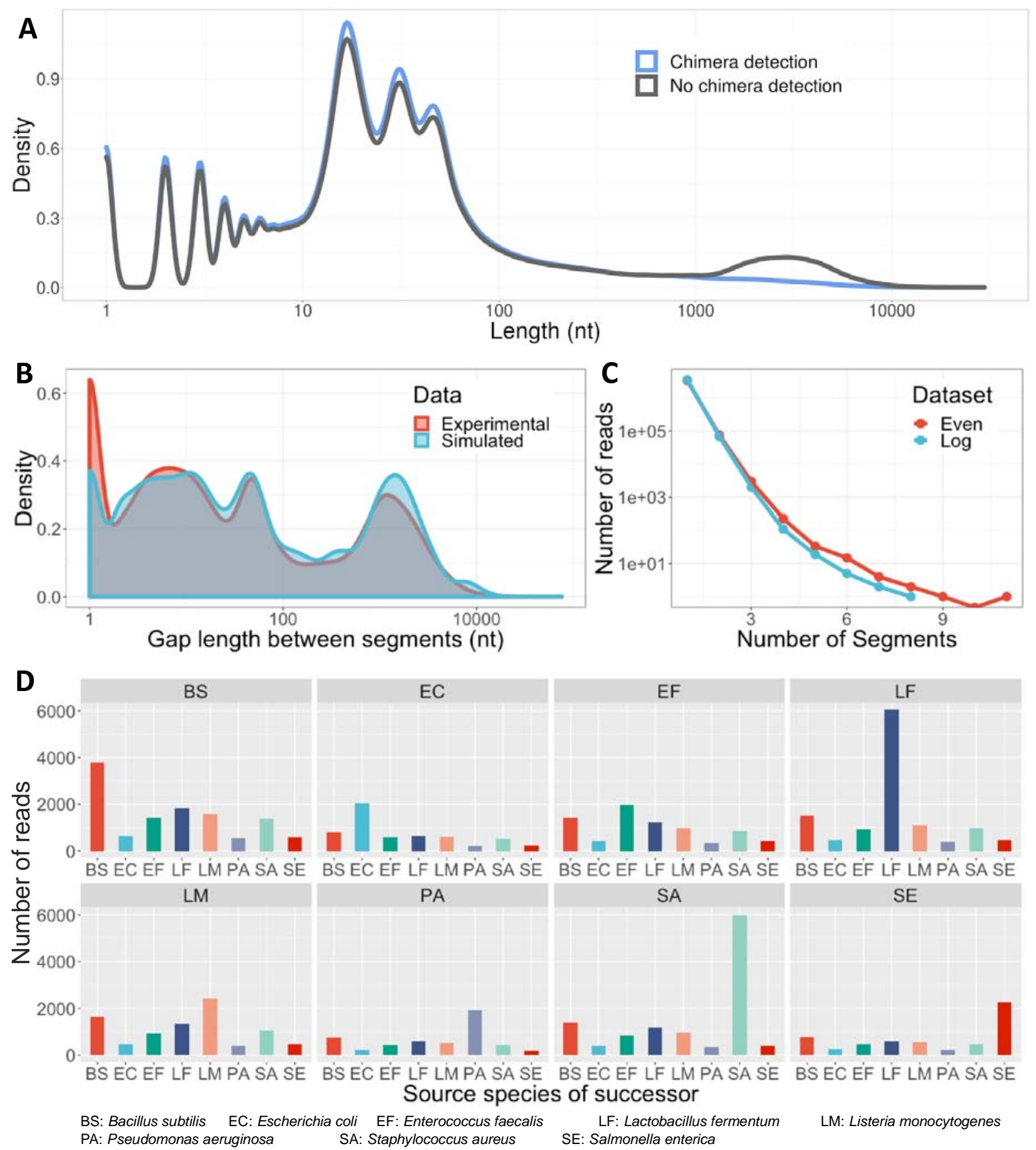

Fig. 2 Chimeric reads detection and simulation. A. The length distribution of the unaligned regions of reads with or without chimeric reads detection for the $\log$ dataset. $\mathbf{B}$. The performance of gap length simulation for the $\log$ dataset. C. The number of segments each read contains for the Even and $\log$ datasets. D. All segments in chimeric reads in the Even dataset are converted into overlapping pairs. Each facet represents one source species of the first segment and the $x$-axis represents the source species of the second segment. Each facet shows the probability of the second segment given the source 
species of the first one. Cryptococcus neoformans and Saccharomyces cerevisiae are excluded here due to their low abundances.

167 Based on the source species to which each split alignment belongs, chimeric reads can be

168 classified as "intra-species-chimeric" or "inter-species-chimeric". It is observed that the source

169 species of the first segment is affected by the abundance level, while subsequent segment is

170 more likely to be influenced by the identity of the previous species (Fig. 2D). We postulate that

171 this is because DNA molecules of the same species are more likely to gather near the nanopore

172 than being homogeneously dispersed in the buffer. Regardless of the actual cause, this

173 phenomenon can be approximated as a simplified hidden Markov model with a generalized

174 emission probability, which is defined as shrinkage rate $s$ here (Methods). To our calculation, $s$

175 is equal to 0.77 for the Even dataset and 0.73 for the Log dataset, suggesting that its value is

176 stable across each dataset.

178 Abundance estimation

179 Existing abundance estimation methods generally quantify the number of mapped reads or $k$ -

180 mers, under the presumption that all reads have equal lengths. However, since the ONT read

181 length varies several folds of magnitude, it is likely that the mean read length for each speces is

182 different. When all species are equally and deeply sequenced, according to central limit

183 theorum, the standard deviation of mean lengths would scale with $1 / \sqrt{n}$, where $\mathrm{n}$ is the

184 number of species. In reality, low-abundant species may have a even higher standard deviation

185 because there are fewer sequences representing it. We observe the mean read lengths of

186 uniquely aligned reads in the $\log$ datasets to range between $3-7 \mathrm{~kb}$, neccesitating base-level

187 instead of read-level quantification algorithms (Fig. S2). One key challenge that confounds 
short-read metagenomic analysis is ambiguously aligned reads. In ONT datasets, however, most reads are long enough to span inter- and intra-species homologous regions, and the chimeric reads detection feature can resolve the estimation for reads that have multiple sub-alignments

191 and for reads that span across the start site of a circular genome. For the remaining small

192 fraction of multi-aligned reads between closely-related species, the estimation for them can be 193 optimized using the EM algorithm (Methods).

195 To benchmark, we compared the performance of four abundance estimation methods: Meta-

196 NanoSim estimation with chimeric reads detection, Salmon quantification with - -meta 197 option (Salmon) (32), the base-level estimation reported in the paper that released the dataset 198 (denoted as "Data Note" from hereon) (8), and MetaMaps. For Meta-NanoSim estimation, we 199 performed an ablation study that removes key components of the algorithm step by step, 200 including estimation on read-level with chimeric reads detection (Meta-NanoSim CR) or with 201 EM algorithm (Meta-NanoSim ER), estimation on base-level (Meta-NanoSim B), base-level with 202 chimeric reads detection (Meta-NanoSim CB), base-level with EM algorithm (Meta-NanoSim EB), 203 and base-level with chimeric reads detection fine-tuned by EM algorithm (Meta-NanoSim ECB). 204 All compared methods, except for MetaMaps, are computed based on Minimap2 alignments. 205 We compared the estimated abundances to the expected ones provided by the manufacturer 206 and computed all the metrics: R-squared, standard deviation, and percent error.

208 In general, all base-level quantification methods performed better than read-level 209 quantification methods (Salmon, MetaMaps, Meta-NanoSim CR and ER), and Meta-NanoSim 
210 base-level estimations have the highest correlation with the expected abundances (Table 1, Fig.

211 S3). For the Even dataset, all four Meta-NanoSim base-level methods performed similarly; the

212 stand-alone base-level quantification has the highest R-squared value for the Even dataset,

213 while the chimeric reads detection helped reduce the percent error, mainly for low-abundance

214 species Cryptococcus neoformans (Fig. S3). MetaMaps, as a read-level quantification method

215 designed specifically for ONT metagenomic data, although ranked highest among this category,

216 showed a big discrepancy compared to base-level methods with a near-doubled percent error.

217 For the Log dataset, Meta-NanoSim base-level estimations also had similar R-squared values,

218 higher than other methods. Since the metrics are close to each other for the Log dataset and

219 the performance on low-abundance species may be overshadowed by high-abundance species,

220 we also computed the coefficient of correlation and error between log-transformed estimated

221 and expected abundances. After log-transformation, Salmon, Meta-NanoSim ECB, and Meta-

222 NanoSim EB showed similar performance with Salmon having the highest correlation and Meta-

223 NanoSim ECB having the lowest percent error. The metrics for Meta-NanoSim estimation

224 without EM, on the other hand, dropped significanly due to difficulties in differentiating multi-

225 mapped reads for low-abundance species. When estimating the abundance levels for the $\log$

226 dataset, Minimap2 incorrectly assigned 18,212 reads to the $E$. faecalis genome as primary

227 alignments, when they can also be aligned to an inter-species homologous region in the $L$.

228 monocytogenes genome. In fact, E. faecalis is a low-abundance species in the Log dataset with

229 only 33 unique alignments. Therefore, the methods with EM algorithm resolved the multi-

230 aligned reads problem, indicating that EM can be advantageous for datasets consisting of

231 similar genomes but with large variances in abundance levels. 
233 To recapitulate our findings, we also chose another logrighmically distributed mock microbial

234 community, denoted as the Adp dataset (33) (Methods), and repeated the quantifications with

235 Meta-NanoSim base-level methods, Salmon, and MetaMaps (Table S1). Similarly, all four Meta-

236 NanoSim methods performed similar to each other with the highest correlation to the expected

237 values. MetaMaps quantification, although had the lowest percent error among all compared

238 methods, showed a much lower correlation in terms of R-squared and standard deviation.

239 Taken all together, Meta-NanoSim base-level quantification after chimeric read detection and

240 fine-tuned by EM algorithm balanced correlation and percent error well, making it preferrable

241 for naturally occurring microbial communities with various abundances.

Table 1 Statistical analysis of the abundance estimation results compared to expected abundances.

\begin{tabular}{|c|c|c|c|c|c|c|c|c|c|c|c|}
\hline \multirow{2}{*}{ Tool } & \multicolumn{4}{|c|}{ Algorithm } & \multicolumn{3}{|c|}{ Even dataset } & \multicolumn{4}{|c|}{ Log dataset } \\
\hline & $\mathbf{E}$ & $\mathrm{C}$ & B & $\mathbf{R}$ & $\mathbf{R}^{2}$ & Std & PE & $\mathbf{R}^{2}$ & $\log \mathbf{R}^{2^{*}}$ & Std* & PE \\
\hline \multirow{6}{*}{$\begin{array}{c}\text { Meta- } \\
\text { NanoSim }\end{array}$} & $\sqrt{ }$ & & $\sqrt{ }$ & & .7463 & 0.0225 & 144.5317 & 1.0000 & 0.9920 & 0.1818 & 256.7856 \\
\hline & & $\sqrt{ }$ & $\sqrt{ }$ & & 0.7465 & 0.0225 & 144.0877 & 0.9999 & 0.7899 & 295 & 53349.95 \\
\hline & $\sqrt{ }$ & & $\sqrt{ }$ & & 8 & 0.0 & 145.6229 & 0 & 17 & & \\
\hline & & & $\sqrt{ }$ & & 0.7499 & 0.0224 & 145.4656 & 1.0000 & 0.7895 & 0.9304 & 3.94 \\
\hline & & $\sqrt{ }$ & & $v$ & 0.4305 & 0.0337 & 326.3432 & 0.9980 & 0.7778 & 0.9560 & 57527.07 \\
\hline & $\sqrt{ }$ & & & $\sqrt{ }$ & 0.4396 & 0.0335 & 313.1466 & 0.9980 & 0.7776 & 0.9565 & 57651.72 \\
\hline Salmon & $\sqrt{ }$ & & & $\sqrt{ }$ & 0.4269 & 0.0 & 502 & 0.9978 & 0.9955 & 66 & 368 \\
\hline Data Note & & & $\sqrt{ }$ & & 0.6702 & 0.0257 & 181.1667 & 0.9 & 0.9863 & 0.2374 & 314 \\
\hline MetaMaps & $\sqrt{ }$ & & & $\sqrt{ }$ & 0.4420 & 0.0334 & 258.4563 & 0.9979 & 0.9652 & 0.3781 & 1366.705 \\
\hline
\end{tabular}

$\mathbf{R}^{2}$ : R-squared, Std: standard deviation, PE: summation of percent error

E: EM algorithm, B: base-level quantification, R: read-level quantification, C: chimeric reads detection

* The expected and estimated abundances are log-transformed before calculating R-squared value and standard deviation.

243 Because of the deviation between expected and estimated abundance levels, we introduce a

244 feature that can simulate this observation. With user-provided deviation range, Meta-NanoSim

245 first varies the abundance levels to desired fluctuation, and then starts the simulation process 
246 (Methods). We compared the deviation between expected abundances and experimental data,

247 and the simulation results of NanoSim and CAMISIM (Fig. 3). The distribution of abundance

248 deviations of experimental data and Meta-NanoSim simulated reads are statistically the same

249 (Kolmogorov-Smirnov test $p$-value $=0.79$ ), while the one of CAMISIM reads is pronouced as it

250 does not provide this feature.

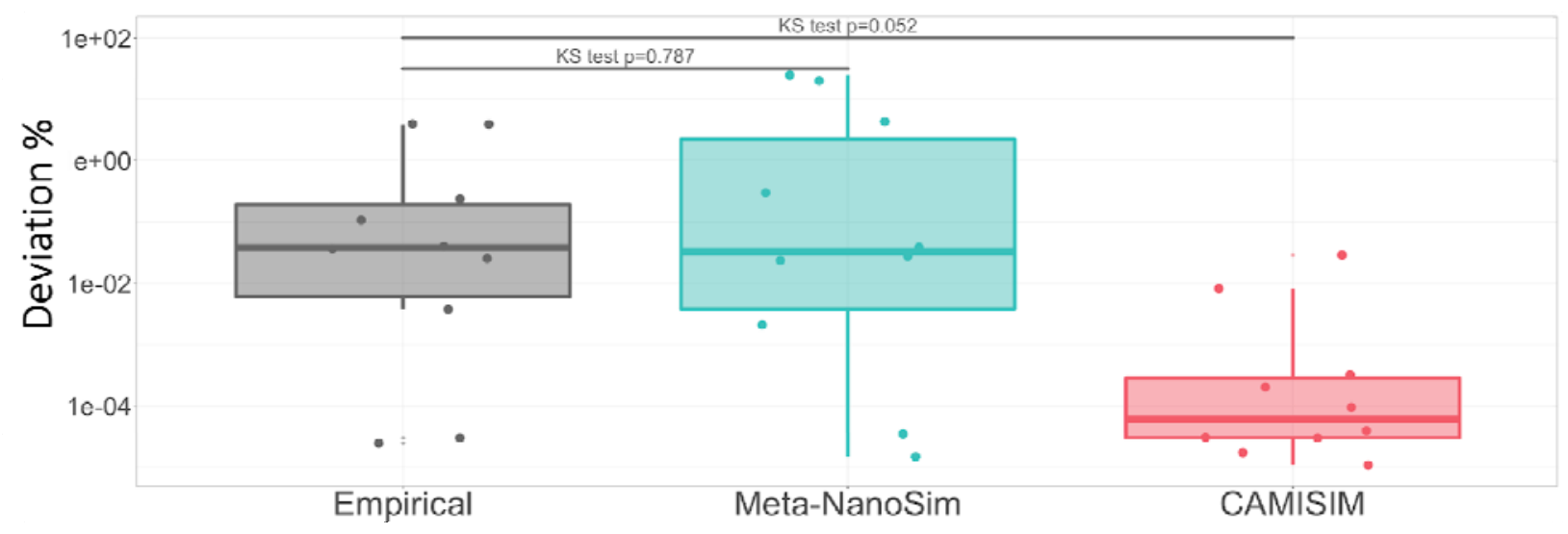

Fig. 3 The simulation of abundance level deviations. In this plot, each dot represents microbial genome, and the $y$-axis represents the deviation in percentage between the expected values and experimental / simulated values.

\section{Comparison between simulated and experimental datasets}

253 To demonstrate the performance of Meta-NanoSim, we trained it with the Log dataset and

254 compared the simulated datasets against the result of CAMISIM. With eight processors,

255 simulation of one million reads took under 20 minutes (or under 160 CPU-minutes) for Meta-

256 NanoSim, while CAMISIM required more than six hours to complete.

258 The read lengths of simulated datasets from Meta-NanoSim follow the empirical length 259 distribution closely, with a median read length peak at 4,040 nt (3,994 nt for empirical reads)

260 (Fig. 4A). In contrast, the lengths of CAMISIM-simulated reads deviate far from those of the 
empirical data. Because CAMISIM is only compatible with an old version of NanoSim and uses

262 hard coded pre-trained models learnt from a genomic sequencing data, it was not possible to

263 test it coupled with Meta-NanoSim. Moreover, the length distribution of unaligned parts on

264 Meta-NanoSim simulated reads captures the patterns in empirical reads well, with multiple

265 peaks below 100 nt. In contrast, the lengths of unaligned part in CAMISIM reads are inflated as

266 it does not detect nor simulate chimeric reads. Both Meta-NanoSim and CAMISIM mimic the

267 mismatch and deletion events well when compared to the empirical dataset (Fig. 4B), which

268 demonstrates the robustness of NanoSim mixture statistical models. However, Meta-NanoSim

269 simulates insertion and match events better than CAMISIM, also due to the change of model.
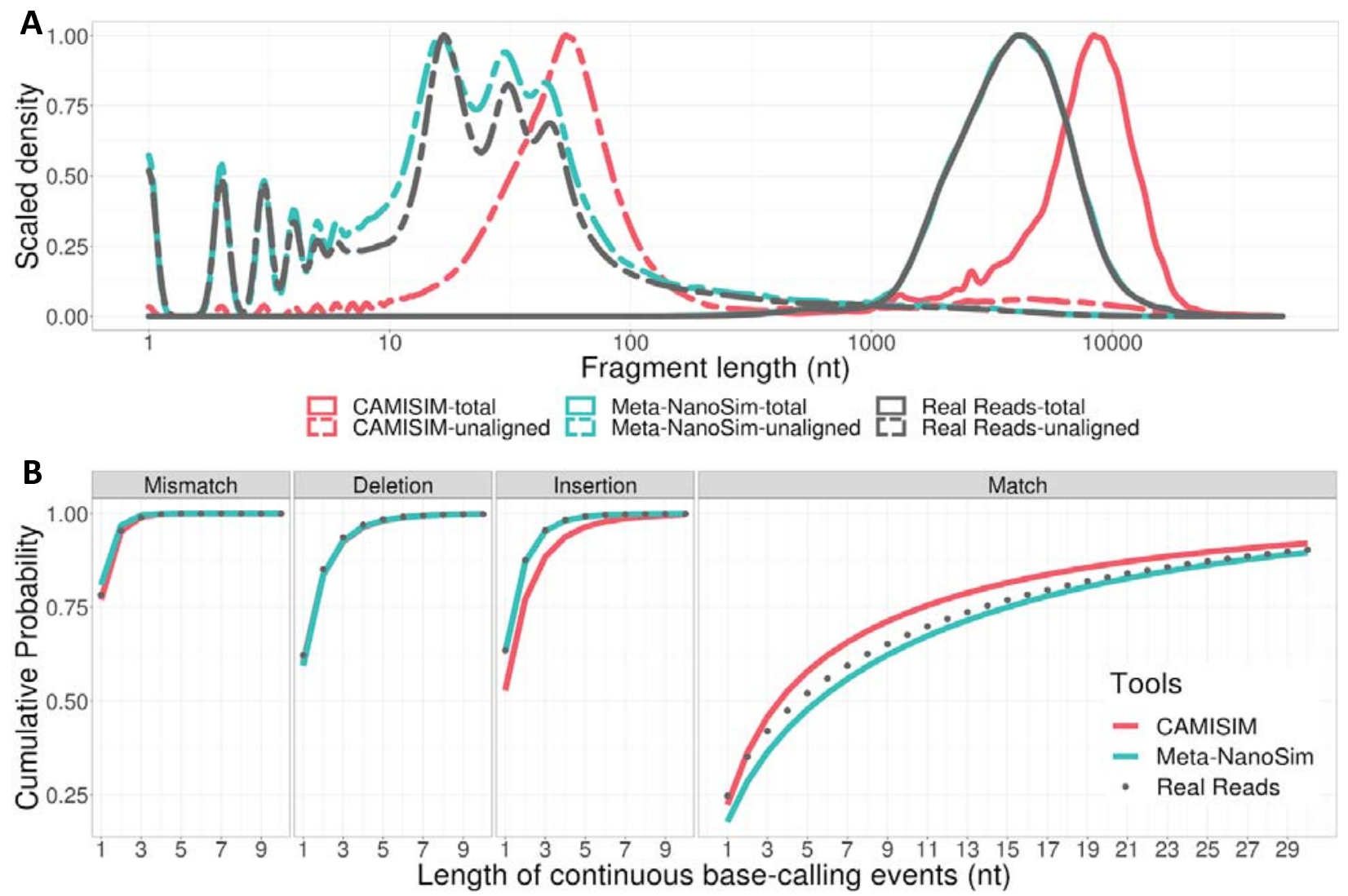

Fig. 4 Performance of Meta-NanoSim and CAMISIM in simulating one million reads from the Log dataset. A. Comparison of read length distributions in the empirical vs. simulated reads. Unaligned length represents the length of unaligned part of each aligned read. B. Cumulative probability function of the lengths of matches/errors in empirical and simulated reads. 
271 In addition, we challenged Meta-NanoSim with two more simulation tasks. First, we simulated

272 two samples at the same time with the pre-trained model from the Log dataset. Each sample

273 contained one million reads from the seven species from the Adp dataset with different

274 abundance levels. Meta-NanoSim simulation finished within 51 min with eight processors.

275 Although the metagenome to be simulated is completely different from the training one,

276 simulated reads exhibited similar read features as the experimental data, and the abundance

277 levels are $100 \%$ in accordance with the expected values (Fig. S4). Next, we randomly picked a

278 saliva sample from the Human Microbiome Project (HMP) and tried to simulate ONT reads

279 using the same microbial composition (34). With 125 different bacteria straines, it took Meta-

280 NanoSim less than three hours to simulate 10 million reads, including the time used for

281 streaming reference genomes from RefSeq server.

283 Benchmarking on assembly tasks

284 Meta-NanoSim simulated reads can be used to benchmark and assess the performance 285 ofbioinformatics algorithms. To demonstrate an application of Meta-NanoSim, we simulated

286 four sets of data with 1, 2, 4, 10 million reads to assess the correctness, scalability and 287 robustness of metaFlye, a long-read metagenomic assembler. We used the models learnt from 288 the $\log$ dataset and the abundance levels of the 10 species are shown in Fig. 5. With 128 289 threads, runtimes ranged from one hour to seven hours (Table S2). The maximum resident set 290 size for 10 million reads dataset was $212 \mathrm{~GB}$, but intermediate files occupied over 10 TB of disk 291 space during consensus-building stage. We also tried to assemble a larger dataset of 20 million 
292 reads, however the assembly failed after 30 days with an out-of-memory error on a 1 TB RAM

293 server. Before completing, the maximum resident set size was $1.75 \mathrm{~TB}$, and the most time-

294 consuming stage was the graph simplifying stage (two weeks).

296 In total, MetaFlye reconstructed 27.81 Mbp sequences, adding up to $43.72 \%$ of the total

297 reference genome for the $4 \mathrm{M}$ reads simulated dataset. These metrics are similar to the

298 reported assemblies using the original training dataset with $3.48 \mathrm{M}$ reads (28.20 Mbp

299 assembled length that covered $46.00 \%$ of the reference metagenome) (29). Generally speaking,

300 the average genome coverage is positively correlated with the number of sequencing reads and

301 abundance levels, and accordingly the genome reconstruction fraction and NGA50 length are

302 positively correlated with the coverage (Fig. 5). As expected, sub-1x coverage genomes have

303 very poor reconstructions. Between $1 x$ and $10 x$, the positive correlation is mirrored in multiple

304 species, including B. subtilis, S. cerevisiae, E. coli and S. enterica. When the coverage reaches

$30510 x$, metaFlye is able to reconstruct the genome to nearly $100 \%$ (S. cerevisiae in the $4 \mathrm{M}$ dataset,

306 Fig. 5). When the coverage reaches 30x, the NGA50 length can cover the whole genome size ( $B$.

307 subtilis in the $2 \mathrm{M}$ dataset). Similarly, the contigs reconstructed for these two species decrease

308 as the number of reads increase, showing how increasing sequencing depth can help

309 assembling into one contig for B. subtilis and nearly one contig per chromosome for $S$.

310 cerevisiae. In contrast, when the coverage is not high enough, the assemblies may be

311 fragmented or even mis-assembled in case of E. coli and S. enterica. However, a higher

312 coverage does not necessarily lead to a better assembly quality. The reconstruction of $L$.

313 monocytogenes deteriorates with more reads when the fold coverage exceeds 1000x. Although 
314 the genome fraction remains $100 \%$, the NGA50 is only half or less of the genome size for the

$3152 \mathrm{M}, 4 \mathrm{M}$, and $10 \mathrm{M}$ datasets. The drop in NGA50 length can be explained by the increasing

316 number of reconstructed contigs and mis-assemblies (Fig. 5). With one million reads, there are

317 only four contigs that can be mapped to the L. monocytogenes genome, and there are no mis-

318 assemblies detected. However, we believe that the higher sequencing depth above $1000 \mathrm{x}$ has

319 led to many more misassemblies, which lowered the assembly's NGA50. 


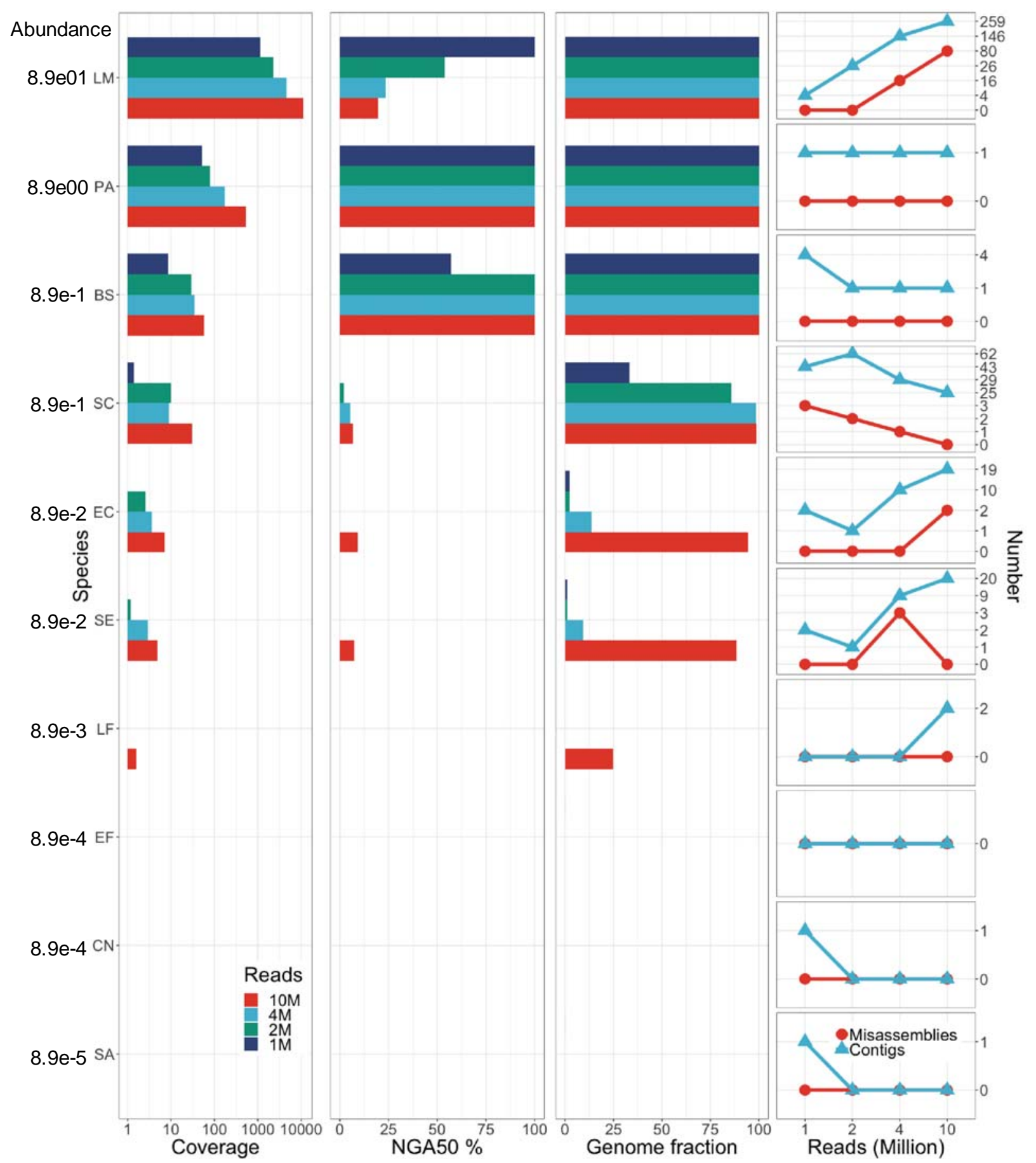

Fig. 5 metaFlye assemblies with four sets of simulated datasets. Four sets of simulated datasets include $1,2,4$ and 10 million reads, respectively. The abundance is the expected abundance level during simulation. Coverage panel is the average read depth including plasmids. NGA50 \% represents the NGA50 length divided by the reference genome size. Genome fraction is a proportion between the assembled sequences and each corresponding genome. The right-most panel shows the number of misassemblies and assembled contigs as the number of simulated reads increases. BS: Bacillus subtilis, CN: Cryptococcus neoformans, EC: Escherichia coli, EF: Enterococcus faecalis, LF: Lactobacillus fermentum, LM: Listeria monocytogenes, PA: Pseudomonas aeruginosa, SA: Staphylococcus aureus, SC: Saccharomyces cerevisiae, SE: Salmonella enterica. 


\section{DISCUSSION}

321 The applications of nanopore sequencing on metagenomic projects are rapidly expanding,

322 motivating the development of metagenomic analysis tools tailored for this specific data type.

323 In this work, we bring two main contributions to ONT metagenomic analysis tasks: (1)

324 introduction of a new base-level quantification method for metagenomic abundance estimation;

325 and (2) an upgrade of NanoSim for metagenomic characterization and simulation.

327 Reference-based metagenomic abundance estimation is key to investigating the microbial 328 composition of an enviroment, and this has been a recurring topic for all emerging sequencing

329 technologies. The long read length of ONT reads provides an opportunity to resolve

330 homologous regions between species or even strains, but other complications arise due to the

331 high error rate and non-uniform read lengths. Existing methods generally assume uniform read

332 lengths and therefore they only need to count the number of mapped reads or $k$-mers.

333 However, it is unreasonable to treat, say, a $100 \mathrm{bp}$ long read and a $1000 \mathrm{bp}$ long read the same

334 when calculating their contributions to the genome abundance. Our results echoed that it is

335 necessary to quantify microbial abundances on a base-level rather than read-level to leverage

336 this data type. In addition to the higher error rate, a small yet substantial fraction of ONT reads

337 are chimeras, which may obscure the accuracy of estimates. The chimeric read detection

338 feature in Meta-NanoSim works by searching for best compatible alignments and helps

339 reducing the percent error in abundance estimation. For multi-aligned segments whose source

340 species cannot be differentiated, we adopted an EM algorithm to optimize their proportional

341 contributions to each potential source species. Our benchmarking results demonstrate that the 
342 combination of these three components can achieve the best correlation with expected

343 abundance. We note that Meta-NanoSim quantification performs better when the abundance

344 levels are more uniform or when low-abundance microbes do not share large homologous

345 regions with high-abundance microbes. Depending on wheather the user wishes to achieve a

346 higher correlation or lower percent error, they can choose to disable or enable chimeric reads

347 detection, respectively. Although our work is limited to reference-based quantification, we

348 expect it to elucidate the design of reference-free methods to have a broader application.

350 Built on top of the abundance estimation, Meta-NanoSim is able to simulate datasets with

351 desired abundance profiles. It can also recapitulate the abundance level deviation from

352 expected values, which is especially useful for designing sequencing projects. When the rough

353 abundance of a microbial community is known, it is essential to know how deep the sequencing

354 needs to be so that each species can be covered by a sufficient number of sequencing reads.

355 However, when the sequenced abundance differs from the expected value, simulated data with

356 abundance variations true to the platform can inform the relationship between sequencing

357 depth and abundance levels.

359 The general workflow of characterization and simulation of Meta-NanoSim follows the same 360 paradigm of the previous versions of NanoSim. The chimeric read detection in characterization

361 stage provides a means to profile all chimeric reads in a library regardless of its root cause.

362 When the reference metagenome is inclusive, the chimeric reads are likely introduced by 363 library preparation and sequencing artifacts; while in reality, since the detection relies on 
364 alignment, some chimeric reads may also be attributed to structural variants when the source

365 genome is not present in the reference. In this case, the output of the characterization stage

366 can be used to further investigate such events with specifically designed statistically models

367 and algorithms. Three main new features for simulation added to Meta-NanoSim are chimeric

368 read simulation, streaming reference genome for online server, and the simulation of a

369 metagenome composed of a mixture of both linear and circular genomes. As chimeric reads

370 may interfere with downstream analyses, simulated datasets with these artifacts are needed

371 for more accurate performance assessment. Characterizing this feature and introducing it to

372 simulated reads will also diversify error types in the reads, helping to improve the robustness of

373 related algorithms. Reference genome streaming is uniquely advantageous when simulating a

374 large metagenome with hundreds of species. It eliminates the reference genome downloading

375 step for users and saves disk space while keeping the runtime reasonable. Similarly, since

376 metagenomes are naturally composed of both linear and circular genome topologies, having a

377 simulated dataset supporting this important characteristic will add credibility to benchmarking

378 results and better forecast performance with experimental data.

380 The benchmarking on metagenomic assembler showcased that Meta-NanoSim can facilitate

381 relevant tool development as well as guide sequencing projects. The resulting assembly quality

382 of Meta-NanoSim simulated reads is comparable to that of the experimental data with similar

383 coverage. Although publicly available mock community sequencing data provide a more

384 realistic training and test set, simulated data provide a ground truth and has no limit in size,

385 making them perfect for testing the accuracy and scalability of algorithms. Through the use of 
simulated datasets, we demonstrated that metaFlye assembler performs best when the species species, it is suggested to calculate the number of reads needed given an estimated abundance reads to achieve 10 -fold coverage for a $0.1 \%$ abundant species with a genome size of $5 \mathrm{Mb}$.

391 When assembling real microbial communities with highly variable abundance levels, we

392 recommend multiple rounds of assembly with different sample sizes to achieve the best 393 performance for both high- and low-abundance microbes. In addition, developers may analyze

394 in depth the mis-assemblies and errors in assembled contigs with the ground truth provided by

395 Meta-NanoSim to improve their algorithms. The effect of chimeric reads, as a common source 396 of mis-assemblies, can be easily evaluated with simulated reads. Meta-NanoSim also has a

397 perfect read simulation feature, which would allow users to learn the relationship between 398 assembly and coverage without interference from read base errors. Furthermore, Meta-

399 NanoSim simulated datasets can serve as suitable training and testing datasets for a wide range 400 of algorithms, including read error correction, taxonomy binning, and compositional estimation.

402 CONCLUSIONS

403 Meta-NanoSim is an ONT metagenomic simulator that simulates complex microbial community 404 with read features true to the platform. Given a training dataset, Meta-NanoSim generates read 405 length distributions, error profiles, and alignment ratio models by default. It also detects 406 chimeric reads and quantifies species abundance levels on demand. Meta-NanoSim is aimed to 407 capture the platform-specific features and can be adopted to profile datasets from any 
408 sequencing chemistry and basecallers. The performance of metagenomic quantification of

409 Meta-NanoSim surpasses the performance of the current state-of-the-art. Chimeric read

410 detection improves the read length modelling and helps to reproduce such feature in simulated

411 reads to challenge metagenomic assemblers, taxonomy binners, and abundance quantification

412 tools. For the simulation part, Meta-NanoSim is the first ONT metagenomic read simulator that

413 can simulate chimeric reads and abundance levels at base-level. It also supports

414 multiprocessing and streamed reference genomes from online servers to speed up simulations

415 when hundreds or thousands of genomes are to be simulated in a microbial community. By

416 comparing simulated reads with empirical datasets, we show that Meta-NanoSim preserves

417 some key characteristics of ONT metagenomic reads well. Further, our metagenomic assembly

418 benchmarks demonstrate the usability and utility of Meta-NanoSim and we expect Meta-

419 NanoSim to have broad utility in helping develop, test and improve upon such applications.

421 METHODS

\section{Datasets}

423 For training and testing meta-NanoSim, we used two publicly available ONT datasets generated

424 on the GridION platform (8). ERR3152364 was sequenced from the Zymo CS Even ZRC190633

425 community, and ERR3152366 was sequenced from the Zymo CSII Log ZRC190842 community.

426 Both communities contain eight bacteria species and two fungi species; the abundance levels

427 are $12 \%$ for each bacterium and $2 \%$ for each fungus for the Even dataset; and the abundance

428 levels for the $\log$ dataset are shown in Fig. 3A. Reads were basecalled using Guppy v2.2.2 GPU

429 basecaller with flip-flop configuration, and adapters were trimmed using Porechop v0.2.4. 
430 Reads were also split when internal adapters were found to eliminate false chimeric reads.

431 Additionally, we used another publicly available ONT dataset, the Adp dataset, from project

432 PRJEB44844. This dataset is composed of three libraries with different mean sequence length:

433 ERR5897838, ERR5903399, and ERR5909878. Reads from this dataset were basecalled during

434 the experiment on the GridION using the MinKNOW software. The abundance levels are $50 \% A$.

435 xylosoxidans, $25 \%$ M. morganii, $12 \%$ L. richardii, $6 \%$ P. aeruginosa, $4 \%$ M. wisconsensis, $2 \% P$.

436 vulgaris and $1 \%$ S. dysgalactiae.

438 The experimental dataset for naturally occurring microbial community is from the HMP project,

439 human saliva sample SRS019120. The abundance table of the sample was downloaded from

440 https://hmpdacc.org/hmp/HMSCP/. In the original file, there were 176 bacteria strains, and

441 after removing the ones without reference genome in RefSeq, we compiled a list of 125 strains

442 with their RefSeq reference genome ftp site. We computed the abundance levels based on the

443 size of the reference genome and the estimated depth in the abundance file, and fed it into

444 Meta-NanoSim for simulation. The simulated 10 million reads are uploaded to Zenodo for

445 academic use: https://doi.org/10.5281/zenodo.5712441.

\section{Chimeric reads detection and simulation}

448 During alignment, one ONT read may have multiple sub-alignments to different loci on the

449 reference genome / metagenome. When the query and reference coordinates of two sub-

450 alignments do not overlap, we define them as compatible alignment of each other. Finding the

451 best compatible alignment set problem is akin to finding the largest compatible interval set in 
452 computer science. For each read, we exhaustively search for all compatible alignments for each

453 sub-alignment to generate a list of compatible alignment sets (Fig. S1). We then select the best

454 element from the list for downstream analysis, based on alignment quality and total aligned

455 length. If, for a given read, the best element contains two or more compatible alignments, the

456 read is considered as chimeric and its aligned length, gap length, and source species (in

457 metagenome mode) are modelled for simulation. One exception here is reads that span the

458 start position of a circular genome. These reads are detected but not considered chimeric. The

459 two sub-alignments are cancatenated as one long alignment to count towards the aligned

460 length and source species.

461

462 To determine the source species for each segment in chimeric reads, we build a simplified

463 hidden Markov model where the start probability is the input abundance, the emission

464 probability represents which species the next segment is coming from given the previsous one,

465 and the transitional probability of species is the change of abundance in the underlying Markov

466 chain. Since species to be simulated, namely the states in a Markov model, may be different

467 between the training and simulation metagenome, we generalize the emission probability as a

468 single value called shrinkage rate $s(0<s<=1)$. This parameter describes the reduction of

469 abundances (probabilities) of other species, while maintaining the relative abundances among

470 them. Assuming the input abundance is $\left\{p_{A}, p_{B}, p_{C}, \ldots, p_{N}\right\}$ for $n$ species, when the first segment

471 comes from species $A$, the transitional probabilities for the other species would become $\left\{s \times p_{B}\right.$,

$\left.472 s \times p_{C} \ldots s \times p_{N}\right\}$ and the transitional probability for A would be inflated as $1-s \times \sum_{i=B}^{N} p_{i}$. To learn

$473 s$, all segments in chimeric reads are divided into overlapping pairs, and the probability for the 
474 source species of the second segment being different from the first one is recorded. In this way,

475 we can calculate the reduction of abundance for every species. The average reduction is the

476 shrinkage rate, and the inflated abundance for being from the same species can be inferred as

477 well. The shrinkage rate can also be adjusted by the user, to 1 for example, if one assumes all

478 DNA molecules are homogeneously suspended in the buffer.

479

480 To summarise the simulation of chimeric reads, Meta-NanoSim first determines the number of

481 segments to be simulated based on the Geometric distribution. If the number of segments is

482 greater than or equal to two, it is a chimeric read. Then, Meta-NanoSim generates the lengths

483 of each segment and gap between them using kernel density estimation learnt from empirical

484 reads. The source species of the first segment is randomly picked based on the input

485 abundance level. Starting from the second segment, the abundance levels are re-computed

486 based on the previous species and $s$. The source species is determined one after another, and

487 then sequences are extracted, mutated with purposely introduced errors, and assembled in the 488 same process as non-chimeric reads.

490 Abundance estimation

491 Meta-NanoSim offers two estimation methods, one with the chimeric reads detection and one

492 without. When chimeric reads detection is enabled, all subalignments are used for computing;

493 when it is disabled, only the primary alignments are used. Meta-NanoSim records the aligned

494 bases for each sub-alignment towards their source genome, and then uses EM algorithm

495 (Supplementary Methods) to assign multi-aligned segments proportionally to their putative 
source genomes iteratively.

498 Abundance deviation simulation

499 Meta-NanoSim offers a means to simulate the abundance deviation with user-defined lower

500 and upper deviation boundaries. We noticed a weak positive correlation between genome size

501 and abundance deviation in our analysis. During simulation we first randomly draw a list of

502 relative error between the deviation boundaries. Next we assign these errors to each genome

503 based on their sizes, namely larger errors are assigned to larger genomes and smaller errors are

504 assigned to smaller genomes. After modifying with errors, the abundances are renormalized to

505 have a total abundance of $100 \%$.

507 Abundance estimation benchmarking

508 For the Data Note estimation, we used the data provided in the paper where the dataset was

509 first reported (8). For all other three methods, we first mapped the reads to the ZymoBIOMICS

510 reference genome version 2 with Minimap 2.17-r941. To run Salmon, we used the - -meta

511 option and --noErrorModel to quantify the abundance for both datasets as suggested in

512 the manual.

\section{Simulation and benchmarking}

515 We trained Meta-NanoSim on the mock community datasets with two options, --chimeric

516 and --quantification (src/read_analysis.py metagenome -i input.fa

517 -gl metagenome_list_for_training -t $12-q-c)$, and then simulated one 
518 million reads with --chimeric (src/simulator.py metagenome -gl

519 metagenome_list_for_simulation -a abundance_for_simulation.tsv -

520 dl dna_type_list.tsv -c models/training -t $12-c)$. The abundance levels

521 simulated are the same as the Log dataset and $A d p$ dataset, when respective metagenome

522 were used for simulation. CAMISIM uses NanoSim as the engine for ONT read simulation. The

523 pre-trained profile is hardcoded in CAMISIM, which is trained on an E. coli dataset with

524 NanoSim version one. To run CAMSIM, we had to choose a most updated compatible version of

525 NanoSim (V2.0.0) that reads the specific format of profiles. Then we mapped the simulated

526 datasets to the reference genomes with Minimap 2.17-r941 to calculate the read length

527 distributions and error distributions, and compared the result with the ones of the

528 experimental data.

530 Assembly benchmarking

531 The assembly benchmarking was performed on a high-performance computing server with 128

532 CPUs and 1 TB memory. We simulated four sets of data with models trained on the Log dataset

533 with default settings and each dataset contains 1, 2, 4, 10 million reads. Then we ran metaFlye

534 2.8.1-b1676 with options --meta, --plasmids, --threads 128 and -g 70m. Next we

535 ran MetaQUAST v5.1.0rc1 with default settings to evaluate the quality of assemblies.

536

537 LIST OF ABBREVIATIONS

538 bp : basepairs

539 EM : Expectation-Maximization 
540 GB : gigabytes

541 GPU : graphics processing unit

$542 \quad M$ : million

543 NGA50 : length of the shortest alignment block for which longer or equal length alignment

544 blocks cover $50 \%$ of the reference genome size

545 nt : nucleotides

546 ONT : Oxford Nanopore Technologies

547 TB : terabytes

548

549 DECLARATIONS

550 Ethics approval and consent to participate

551 Not applicable

552

553 Consent for publication

554 Not applicable

555

556 Availability of data and material

557 Meta-NanoSim is implemented in Python within the NanoSim suite. The source code and pre-

558 trained models used in this study are available on Github: https://github.com/bcgsc/NanoSim.

559 NanoSim version 3.0.2 is used for this work. Meta-NanoSim is platform independent, and is 560 released under the GNU GPL license. 


\section{Competing interests}

563 The authors decare that they have no competing interests.

565 Funding

566 This work was supported by Genome Canada and Genome BC [281ANV]; and by the National

567 Human Genome Research Institute of the National Institutes of Health [R01HG007182].

568 Scholarship funding was provided by the University of British Columbia, and the Natural

569 Sciences and Engineering Research Council of Canada. The content is solely the responsibility of

570 the authors and does not necessarily represent the official views of the funding organizations.

571

\section{Authors' contributions}

573 IB and CY conceived and designed the study. CY designed and implemented the software with

574 the help of $\mathrm{TL}, \mathrm{SH}$, and $\mathrm{KMN}$. KMN and $\mathrm{SH}$ provided additional help with the software

575 maintainence. CY drafted the manuscript, and all authors were involved in its revision. All

576 authors read and approved the final manuscript.

577

578 Acknowledgements

579 Not Applicable

580

\section{REFERENCE}

582 1. Handelsman J. Metagenomics: Application of Genomics to Uncultured Microorganisms. Microbiol 583 Mol Biol Rev. 2004;

$5842 . \quad$ Chen K, Pachter L. Bioinformatics for whole-genome shotgun sequencing of microbial 
communities. PLoS Computational Biology. 2005.

586 3. Schulz F, Alteio L, Goudeau D, Ryan EM, Yu FB, Malmstrom RR, et al. Hidden diversity of soil giant viruses. Nat Commun. 2018;

588 4. Guthrie L, Gupta S, Daily J, Kelly L. Human microbiome signatures of differential colorectal cancer drug metabolism. npj Biofilms Microbiomes. 2017;

$590 \quad 5 . \quad$ Wirbel J, Pyl PT, Kartal E, Zych K, Kashani A, Milanese A, et al. Meta-analysis of fecal metagenomes reveals global microbial signatures that are specific for colorectal cancer. Nat Med. 2019;

593 6. Quince C, Walker AW, Simpson JT, Loman NJ, Segata N. Shotgun metagenomics, from sampling to analysis. Nature Biotechnology. 2017.

7. Brown BL, Watson M, Minot SS, Rivera MC, Franklin RB. MinIONTM nanopore sequencing of environmental metagenomes: A synthetic approach. Gigascience. 2017;

8. Nicholls SM, Quick JC, Tang S, Loman NJ. Ultra-deep, long-read nanopore sequencing of mock microbial community standards. Gigascience. 2019;

9. Fu S, Wang A, Au KF. A comparative evaluation of hybrid error correction methods for errorprone long reads. Genome Biol. 2019;

10. Payne A, Holmes N, Rakyan V, Loose M. Bulkvis: A graphical viewer for Oxford nanopore bulk FAST5 files. Bioinformatics. 2019;

11. Charalampous T, Kay GL, Richardson H, Aydin A, Baldan R, Jeanes C, et al. Nanopore

12. Kafetzopoulou LE, Pullan ST, Lemey P, Suchard MA, Ehichioya DU, Pahlmann M, et al. Biotechnol. 2019; ). 2019;

13. Chan JFW, Yuan S, Kok KH, To KKW, Chu H, Yang J, et al. A familial cluster of pneumonia associated with the 2019 novel coronavirus indicating person-to-person transmission: a study of a family cluster. Lancet. 2020;

14. Greninger AL, Naccache SN, Federman S, Yu G, Mbala P, Bres V, et al. Rapid metagenomic identification of viral pathogens in clinical samples by real-time nanopore sequencing analysis. Genome Med. 2015;

15. Yang C, Chu J, Warren RL, Birol I. NanoSim: Nanopore sequence read simulator based on statistical characterization. Vol. 6, GigaScience. 2017.

16. Hafezqorani S, Yang C, Lo T, Nip KM, Warren RL, Birol I. Trans-NanoSim characterizes and simulates nanopore RNA-sequencing data. Gigascience. 2020;

17. Buck D, Weirather JL, de Cesare M, Wang Y, Piazza P, Sebastiano V, et al. Comprehensive comparison of Pacific Biosciences and Oxford Nanopore Technologies and their applications to transcriptome analysis. F1000Research. 2017; 
19. Lu J, Breitwieser FP, Thielen P, Salzberg SL. Bracken: Estimating species abundance in metagenomics data. PeerJ Comput Sci. 2017;

20. White R, Pellefigues $\mathrm{C}$, Ronchese F, Lamiable O, Eccles D. Investigation of chimeric reads using the MinION. F1000Research. 2017;

628 21. Martin S, Leggett RM. Alvis: a tool for contig and read ALignment VISualisation and chimera detection. BMC Bioinformatics. 2021;

630 22. Marijon P, Chikhi R, Varré JS. Yacrd and fpa: Upstream tools for long-read genome assembly. Bioinformatics. 2020;

23. Xu Y, Lewandowski K, Lumley S, Pullan S, Vipond R, Carroll M, et al. Detection of viral pathogens with multiplex nanopore MinION sequencing: Be careful with cross-Talk. Front Microbiol. 2018;

634 24. Tvedte ES, Gasser M, Sparklin BC, Michalski J, Hjelmen CE, Johnston JS, et al. Comparison of longread sequencing technologies in interrogating bacteria and fly genomes. $\mathrm{G} 3$ Genes | Genomes | Genetics. 2021;

25. Wick RR, Judd LM, Holt KE. Deepbinner: Demultiplexing barcoded Oxford Nanopore reads with deep convolutional neural networks. PLoS Comput Biol. 2018;

639 26. Dilthey AT, Jain C, Koren S, Phillippy AM. Strain-level metagenomic assignment and compositional estimation for long reads with MetaMaps. Nat Commun. 2019;

27. Jia B, Xuan L, Cai K, Hu Z, Ma L, Wei C. NeSSM: A Next-Generation Sequencing Simulator for Metagenomics. PLoS One. 2013;

643 28. Fritz A, Hofmann P, Majda S, Dahms E, Dröge J, Fiedler J, et al. CAMISIM: Simulating metagenomes and microbial communities. Microbiome. 2019;

29. Kolmogorov M, Bickhart DM, Behsaz B, Gurevich A, Rayko M, Shin SB, et al. metaFlye: scalable

30. O'Leary NA, Wright MW, Brister JR, Ciufo S, Haddad D, McVeigh R, et al. Reference sequence (RefSeq) database at NCBI: Current status, taxonomic expansion, and functional annotation. Nucleic Acids Res. 2016;

31. Howe KL, Achuthan P, Allen J, Allen J, Alvarez-Jarreta J, Ridwan Amode M, et al. Ensembl 2021.

652 32. Patro R, Duggal G, Love MI, Irizarry RA, Kingsford C. Salmon provides fast and bias-aware

33. Martin S, Heavens D, Lan Y, Horsfield S, Clark MD, Leggett RM. Nanopore adaptive sampling: a tool for enrichment of low abundance species in metagenomic samples. bioRxiv. 2021; 